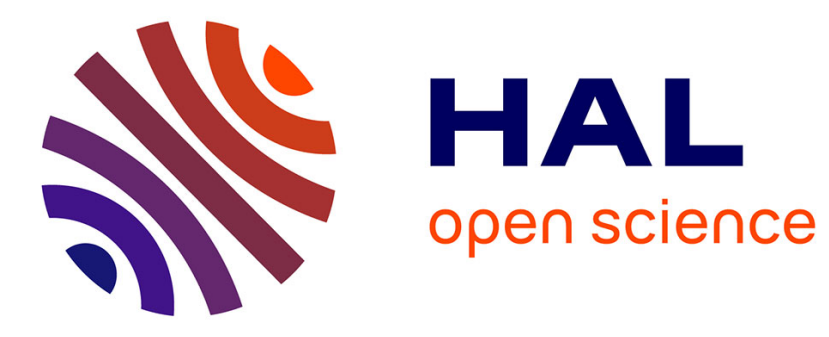

\title{
Détermination expérimentale des paramètres des transistors MOS
}

P. Rossel, Henri Tranduc, Jean-Louis Sanchez, A. Bellaouar

\section{To cite this version:}

P. Rossel, Henri Tranduc, Jean-Louis Sanchez, A. Bellaouar. Détermination expérimentale des paramètres des transistors MOS. Revue de Physique Appliquée, 1983, 18 (8), pp.487-493. 10.1051/rphysap:01983001808048700 . jpa-00245110

\section{HAL Id: jpa-00245110 https://hal.science/jpa-00245110}

Submitted on 1 Jan 1983

HAL is a multi-disciplinary open access archive for the deposit and dissemination of scientific research documents, whether they are published or not. The documents may come from teaching and research institutions in France or abroad, or from public or private research centers.
L'archive ouverte pluridisciplinaire HAL, est destinée au dépôt et à la diffusion de documents scientifiques de niveau recherche, publiés ou non, émanant des établissements d'enseignement et de recherche français ou étrangers, des laboratoires publics ou privés. 


\title{
Détermination expérimentale des paramètres des transistors MOS (*)
}

\author{
P. Rossel, H. Tranduc, J. L. Sanchez et A. Bellaouar \\ Laboratoire d'Automatique et d'Analyse des Systèmes du C.N.R.S., \\ 7, avenue du Colonel-Roche, 31400 Toulouse, France
}

(Reçu le 22 février 1983, révisé le 10 mai, accepté le 13 mai 1983)

\begin{abstract}
Résumé. - On propose des méthodes expérimentales propres à déterminer les paramètres des transistors MOS : longueur effective du canal, mobilité des porteurs, potentiel transversal de réduction de mobilité, résistances en série dans la source et le drain. Elles sont basées d'une part sur la mesure des conductances de sortie à faible tension drain-source effectuée sur des transistors MOS de même géométrie mais ayant des longueurs de canal différentes et, d'autre part, sur la comparaison des caractéristiques de transfert courant-tension en régime de non-pincement, en configuration normale et en configuration de source et drain inversés.
\end{abstract}

Abstract. - We present experimental methods to determine the MOS transistor parameters : effective channel. length, carrier mobility, mobility roll-off coefficient, series drain and source resistances. They are deduced from i) the output conductance measurement, at low drain bias, on a set of MOS transistors with different channel length, and ii) the comparison of the transfer characteristics in the normal and in the reverse configuration, i.e. by inversion of the drain and source electrodes.

\section{Introduction.}

Dans les transistors MOS des nouvelles générations, à canal court (H.MOS) vertical (V.MOS) ou réalisé par double diffusion (D.MOS); les caractéristiques électriques statiques dépendent d'une part de paramètres physiques - mobilité des porteurs, coefficient de réduction de mobilité, dopage du substrat -, géométriques - longueur et largeur du canal, épaisseur d'oxyde de grille -, et d'autre part des valeurs de résistances "d'accès " à la source et au drain. Le poids de celles-ci peut être important devant la résistance du canal lorsque, dans les technologies microniques ou submicroniques, il est fait appel pour prendre les contacts, à des matériaux à résistivité élevée tels que les métaux réfractaires ou les siliciures. De même, lorsque le transistor est prévu pour fonctionner en haute tension et utilise un drain à faible dopage (V.MOS, T.MOS, HEXFET, SIPMOS, ...) la résistance de la zone "drift» de drain est supérieure à celle du canal actif.

Pour déterminer certains paramètres précédents,

$\left({ }^{*}\right)$ Ce travail est supporté par le Contrat 82.A.0290 du Ministère de la Recherche et de l'Industrie. plusieurs méthodes ont été proposées récemment dans la littérature [1-5]. Elles sont pour la plupart basées sur les mesures de la conductance de sortie d'un ou plusieurs composants de différentes longueurs de canal, puis sur un traitement mathématique consistant à identifier ces valeurs expérimentales à celles données par une expression théorique de la conductance. En essayant d'appliquer ces méthodes aux diverses structures de transistors, il est apparu d'une part que certaines de ces techniques sont trop approchées et ne sont pas applicables à toutes les géométries de transistors MOS, d'autre part que d'autres sont incomplètes et ne fournissent que certains des paramètres, et enfin que les mises en ouvre proposées, du moins en ce qui concerne les méthodes qui apparaissent comme étant les plus rigoureuses, sont difficiles d'emploi et délicates d'utilisation. Aucune de ces méthodes ne permet toutefois d'obtenir séparément les valeurs de résistances de drain et de source.

Nous proposons une méthodologie permettant d'obtenir tous les paramètres cités précédemment. Les techniques sont systématiques, simples, adaptées à tous les types de structures. Nous les illustrerons par des exemples d'applications à des transistors MOS plans à source et drain diffusés ou à des structures verticales V.MOS réalisées par double diffusion. 
1. Rappel des équations du transistor en régime ohmique.

Par rapport aux théories classiques, la prise en compte de l'effet des résistances en série avec le drain et la source se traduit, dans le calcul du courant de drain, par une modification des bornes d'intégration sur la variable $\Xi$ qui représente en potentiel l'écart des pseudo-niveaux de Fermi en tout point du canal. Ce courant $I_{\mathrm{D}}$ s'exprime alors en zone ohmique par [10] :

$$
\begin{aligned}
I_{\mathrm{D}}= & \frac{\mu_{0} Z L^{-1} C_{\mathrm{ox}} \psi}{1+\frac{V_{\mathrm{D}}^{\prime}-V_{\mathrm{R}}^{\prime}}{L E_{0}}}\left[V_{\mathrm{D}}^{\prime}-V_{\mathrm{R}}^{\prime}+\psi \cdot \log \frac{\psi+V_{\mathrm{G}}^{\prime}-2 \phi_{\mathrm{F}}-V_{\mathrm{D}}^{\prime}}{\psi+V_{\mathrm{G}}^{\prime}-2 \phi_{\mathrm{F}}-V_{\mathrm{R}}^{\prime}}+\right. \\
+ & \left.2 \sqrt{\phi_{\mathrm{B}}} \cdot\left[\sqrt{V_{\mathrm{D}}^{\prime}+2 \phi_{\mathrm{F}}}-\sqrt{V_{\mathrm{R}}^{\prime}+2} \phi_{\mathrm{F}}-\sqrt{V_{\mathrm{G}}^{\prime}+\psi} \cdot\left(\operatorname{Argth} \sqrt{\frac{V_{\mathrm{D}}^{\prime}+2 \phi_{\mathrm{F}}}{V_{\mathrm{G}}^{\prime}+\psi}}-\operatorname{Argth} \sqrt{\frac{V_{\mathrm{R}}^{\prime}+2 \phi_{\mathrm{F}}}{V_{\mathrm{G}}^{\prime}+\psi}}\right)\right]\right],
\end{aligned}
$$

avec

$$
\begin{aligned}
& V_{\mathrm{D}}^{\prime}=V_{\mathrm{D}}-R_{\mathrm{D}} I_{\mathrm{D}}, \\
& V_{\mathrm{R}}^{\prime}=V_{\mathrm{R}}+R_{\mathrm{S}} I_{\mathrm{D}} .
\end{aligned}
$$

Dans ce formalisme, la référence des potentiels est prise au substrat du-transistor schématisé sur la figure 1. La tension $V_{\mathrm{D}}$ représente la tension externe appliquée entre plot de contact de drain et substrat, $V_{R}$ la tension externe appliquée entre plot de source et substrat, la tension $V_{\mathrm{D}}^{\prime}$ est la tension interne développée au droit de la jonction métallurgique drainsubstrat et $V_{R}^{\prime}$ la tension interne développée au droit de la jonction métallurgique source-substrat. $V_{G}^{\prime}$ est la tension effective de grille. Les variables qui figurent dans cette relation sont respectivement : $Z$ la largeur du canal, $L$ sa longueur effective c'est-àdire la distance séparant les jonctions métallurgiques de source et de drain, $C_{\text {ox }}$ la capacité d'oxyde de grille par unité de surface, $\psi$ le potentiel de réduction de mobilité dû au champ transversal (souvent noté $\theta^{-1}$ dans la littérature), $E_{0}$ le champ critique de mobilité dans la direction longitudinale, $\mu_{0}$ la mobilité des porteurs à faible champ électrique, $R_{\mathrm{S}}$ et $R_{\mathrm{D}}$ les résis-

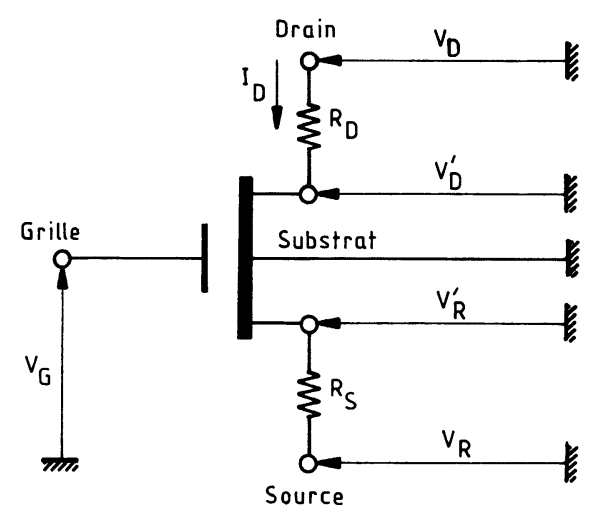

Fig. 1. - Représentation schématique du composant. Définition des potentiels.

[Schematic representation of a biased MOS transistor.] tances internes "d'accès " à la source et au drain, $\phi_{\mathrm{F}}$ le potentiel de Fermi du substrat et $\phi_{\mathbf{B}}$ le potentiel " interne ». Ces deux potentiels sont respectivement définis par :

$$
\begin{aligned}
& \phi_{\mathrm{F}}=\frac{k T}{q} \cdot \log \frac{N_{\mathrm{A}}}{n_{\mathrm{i}}} . \\
& \phi_{\mathrm{B}}=2 q N_{\mathrm{A}} \varepsilon_{0} \varepsilon_{\mathrm{Si}} C_{\mathrm{ox}}^{-2} .
\end{aligned}
$$

Dans ces relations, $q$ est la charge électronique, $n_{\mathrm{i}}$ la concentration intrinsèque, $k T / q$ le potentiel thermodynamique et $\varepsilon_{0} \varepsilon_{\mathrm{Si}}$ la constante diélectrique du silicium, $N_{\mathrm{A}}$ le dopage.

Rappelons pour mémoire que, dans l'expression du courant, la mobilité effective locale des porteurs en fonction des composantes normale et transversale est exprimée sous la forme :

$$
\mu_{\text {eff }}=\frac{\mu_{0}}{1+\left(V_{G}^{\prime}-\Xi(y)-2 \phi_{\mathrm{F}}\right) / \psi} \cdot \frac{1}{1+\left|E_{y}\right| / E_{0}},
$$

où $E_{y}$ est la composante du champ électrique dans la direction $y$, source-drain, au point d'abscisse $y$. Par ailleurs, la tension effective de grille $V_{G}^{\prime}$ tient compte de la différence des travaux de sortie $\phi_{M S}$ entre le métal de grille et le semiconducteur, des charges parasites fixes d'oxyde et de la charge des états de surface sous la forme d'une charge globale $Q_{\text {ss }}$ qui est ramenée à l'interface silice-silicium.

$$
V_{\mathrm{G}}^{\prime} \equiv V_{\mathrm{G}}+\frac{Q_{\mathrm{ss}}}{C_{\mathrm{ox}}}-\phi_{\mathrm{MS}} \text {. }
$$

Le courant de drain précédent s'annule théoriquement pour une valeur particulière notée $V_{\mathrm{T}}$ de la tension grille-substrat, appelée tension de seuil, qui vaut :

$$
\begin{aligned}
V_{\mathrm{T}}=-\frac{Q_{\mathrm{ss}}}{C_{\mathrm{ox}}}+\phi_{\mathrm{MS}}+2 \phi_{\mathrm{F}} & +V_{\mathrm{R}}^{\prime}+ \\
& +\sqrt{\phi_{\mathrm{B}} \cdot\left(V_{\mathrm{R}}^{\prime}+2 \phi_{\mathrm{F}}\right)} .
\end{aligned}
$$


Enfin, rappelons que la conductance de sortie $S_{0}$ à tension drain-source nulle déduite de (1) est égale à :

$$
\left.S_{0}\right|_{V_{\mathbf{D}} \rightarrow V_{\mathbf{R}}}=\frac{\mu_{0} Z L^{-1} C_{\mathrm{ox}} \psi\left(V_{\mathrm{G}}-V_{\mathrm{T}}\right)}{V_{\mathrm{G}}^{\prime}+\psi-2 \phi_{\mathrm{F} .}+\mu_{0} Z L^{-1} C_{\mathrm{ox}} \psi\left(R_{\mathrm{S}}+R_{\mathrm{D}}\right)\left(V_{\mathrm{G}}-V_{\mathrm{T}}\right)},
$$

relation qui peut s'écrire, lorsque source et substrat sont en court-circuit :

$$
\left.\frac{V_{\mathrm{G}}-V_{\mathrm{T}}}{S_{0}}\right|_{V_{\mathrm{D}} \rightarrow 0} ^{V_{\mathrm{R}}=0}=\left[\left(\mu_{0} Z L^{-1} C_{\mathrm{ox}} \psi\right)^{-1}+R_{\mathrm{S}}+R_{\mathrm{D}}\right] \cdot\left(V_{\mathrm{G}}-V_{\mathrm{T}}\right)+\frac{\psi+\sqrt{2 \phi_{\mathrm{F}} \phi_{\mathrm{B}}}}{\mu_{0} Z L^{-1} C_{\mathrm{ox}} \psi} .
$$

\section{Détermination des paramètres.}

Les paramètres que nous avons à déterminer sont :

- Les distances $Z, L$ et $x_{0}$ et par suite la capacité $C_{\mathrm{ox}}=\varepsilon_{0} \varepsilon_{\mathrm{ox}} / x_{0}$. A ce propos, rappelons que la longueur effective $L$ est égale à l'interdistance $L_{\mathrm{m}}$ entre bords internes du masque d'ouverture des diffusions (ou implantation) de source et de drain à laquelle on retranche le double de la somme de la diffusion latérale $x_{1}$ et de la tolérance de surgravure $s$ (Fig. 2) :

$$
L=L_{m}-2\left(x_{1}+s\right) \text {. }
$$

- Le dopage $N_{\mathrm{A}}$ et par suite, les potentiels $\phi_{\mathrm{F}}$ et $\phi_{\mathrm{B}}$ ou inversement,

- le potentiel parasite $\frac{Q_{\mathrm{ss}}}{C_{\mathrm{ox}}}-\phi_{\mathrm{MS}}$, les potentiels $\psi$ et $L E_{0}$,

- la mobilité $\mu_{0}$, les résistances $R_{\mathrm{S}}$ et $R_{\mathrm{D}}$.

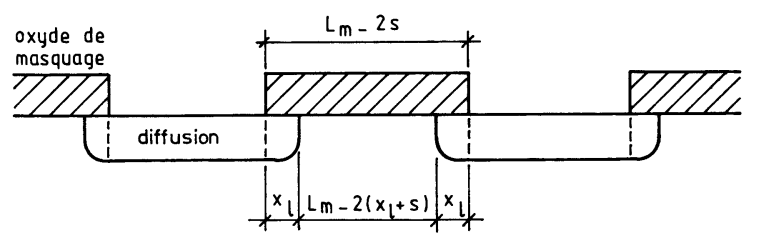

Fig. 2. - Coupe du transistor. Définition des paramètres géométriques.

[Cross-section of a MOS transistor. Definition of the geometrical dimensions.]

Rappelons cependant qu'il est bien établi que certains paramètres peuvent être déterminés par des méthodes éprouvées. Le dopage $N_{\mathrm{A}}$, l'épaisseur $x_{0}$, le potentiel $\frac{Q_{\mathrm{ss}}}{C_{\mathrm{ox}}}-\phi_{\mathrm{Ms}}$ sont obtenus i) par mesure capacité-tension sur des capacités MOS-témoin [6] ou bien ii) par mesure des évolutions de la tension de seuil en fonction de la tension source-substrat [7]. La distance $Z$ - du moins pour les transistors classiques non étroits [11] - est imposée par la géométrie du masque de grille. Par ailleurs, il est aussi connu que le potentiel $L E_{0}$, dont l'effet ne se manifeste qu'à forte tension de drain, n'est pas susceptible d'être mesuré par technique directe. Ainsi, nous nous contenterons de décrire, par la suite, les méthodes propres à obtenir les paramètres restants $\psi, \mu_{0}, L, R_{\mathrm{s}}$ et $R_{\mathrm{D}}$.
2.1 LA MOBILITÉ $\mu_{0}$ ET LA LONGUEUR $L$. - Le principe de mesure de $\mu_{0}$ et $L$ est basé sur les tracés des caractéristiques de sortie de plusieurs transistors ayant des distances source-drain, sur masques, différentes. Lorsque la source et le substrat sont en courtcircuit, que la tension drain-source tend vers zéro $\left(V_{\mathrm{D}}=10 \mathrm{mV}\right.$ par exemple) et que la tension de grille $V_{\mathrm{G}}$ tend vers la tension de seuil $V_{\mathrm{T}}$, la forme limite du courant devient :

$$
\begin{aligned}
\left.I_{\mathbf{D}}\right|_{\substack{V_{\mathbf{D}} \rightarrow 0 \\
V_{\mathbf{G}} \rightarrow V_{\mathbf{T}}}}=\mu_{0} Z L^{-1} & C_{\mathrm{ox}}\left(V_{\mathbf{G}}-V_{\mathrm{T}}\right) \cdot V_{\mathbf{D}} \times \\
& \times\left(1+\sqrt{2 \phi_{\mathrm{F}} \phi_{\mathrm{B}}} / \psi\right)^{-1},
\end{aligned}
$$

forme qui, compte tenu de l'ordre de grandeur du terme $\sqrt{2 \phi_{\mathrm{F}} \cdot \phi_{\mathrm{B}}} / \psi$, peut, pour la plupart des transistors, s'écrire :

$$
\left.I_{\mathrm{D}}\right|_{\substack{V_{\mathrm{D}} \rightarrow 0 \\ V_{\mathbf{G}} \rightarrow V_{\mathbf{T}}}}=\mu_{0} Z L^{-1} C_{\mathrm{ox}}\left(V_{\mathrm{G}}-V_{\mathrm{T}}\right) \cdot V_{\mathrm{D}}
$$

Il apparaît que le terme $\mu_{0} Z L^{-1} C_{\text {ox }}$ représente donc la pente au voisinage du seuil, des variations de la conductance de sortie $I_{\mathrm{D}} / V_{\mathrm{D}}$ en fonction de la tension de grille. La figure 3 traduit la détermination de ce terme sur un transistor plan. La tension de seuil $V_{\mathrm{T}}$ y est aussi mise en évidence.

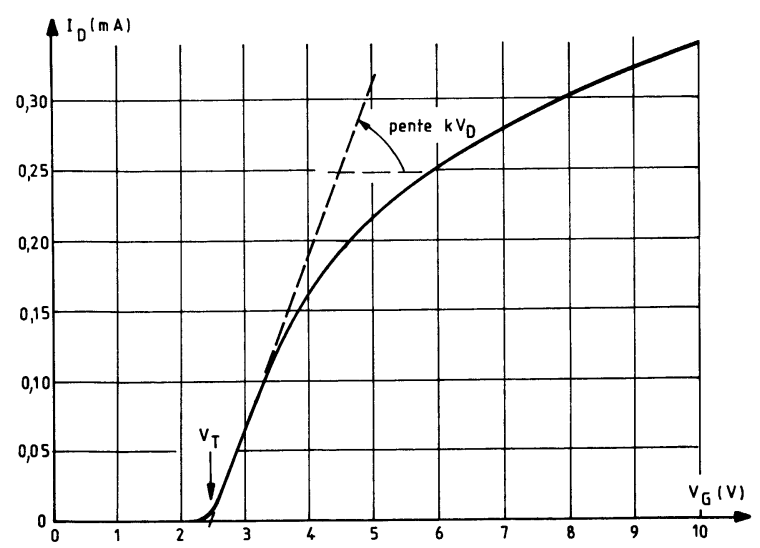

Fig. 3. - Caractéristique de transfert du transistor à faible tension drain-source. Détermination de $V_{\mathrm{T}}$ et $k-$ Mono V.MOS à grille en polysilicium. $V_{\mathrm{D}}=0,1 \mathrm{~V}(L=2 \mu \mathrm{m}$; $\left.Z=200 \mu \mathrm{m} ; x_{0}=1500 \AA ; N_{\mathrm{A}} \simeq 3 \times 10^{16} \mathrm{~cm}^{-3}\right)$.

[Transfer characteristic at low drain bias. Determination of the threshold voltage $V_{\mathrm{T}}$ and of the coefficient $k$.] 
Sur les $i$ transistors, qui ne diffèrent que par la longueur $L_{\mathrm{m} i}$ d'ouverture d'interdiffusion sur masques, on mesure les coefficients :

$$
k_{i} \equiv \mu_{0} \cdot Z \cdot L_{i}^{-1} \cdot C_{\mathrm{ox}},
$$

qui, pour chacun d'entre eux, s'expriment par :

$$
k_{i}=\mu_{0} Z C_{\mathrm{ox}} \cdot\left(L_{\mathrm{m} i}-2 x_{1}-2 s\right)^{-1} .
$$

Le tracé des variations expérimentales de $1 / k_{i}$ en fonction de $L_{\mathrm{m} i}$ (Fig. 4) fournit, par mesure de la pente de la droite obtenue, la valeur $\left(\mu_{0} Z C_{\mathrm{ox}}\right)^{-1}$ et par mesure de l'abscisse à l'origine, la valeur du terme $2\left(x_{1}+s\right)$ qui représente le raccourcissement du canal. On calcule ensuite la valeur de la mobilité $\mu_{0}$ puis les longueurs effectives $L_{i}$ de chaque transistor par la relation 11 .

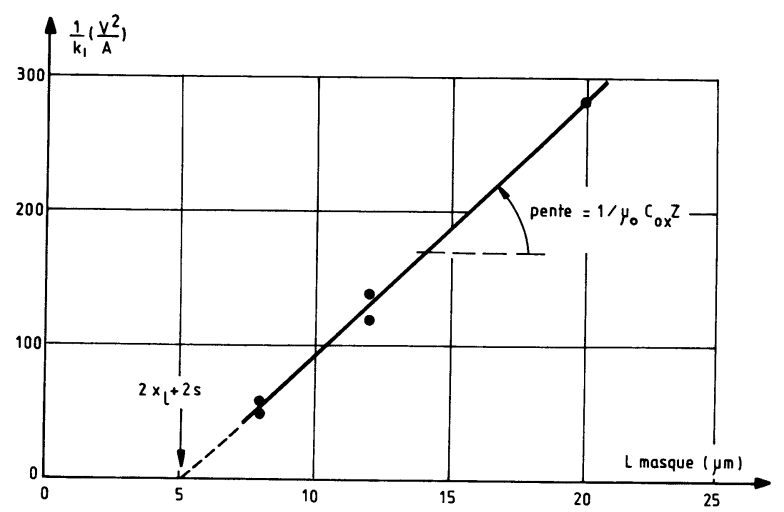

Fig. 4. - Variations du coefficient $k_{i}^{-1}$ en fonction de la longueur sur masque $L_{\mathrm{m}}$. Détermination de $\left(x_{1}+s\right)$ Transistors MOS plan à jonctions diffusées à grille en aluminium $\left(Z=2 \mathrm{~mm} ; x_{0}=1000 \AA, N_{\mathrm{A}}=1,5 \times 10^{16} \mathrm{~cm}^{-3}\right)$

[Experimental variations of the coefficient $1 / k_{i}$ as a function of the channel length $L_{\mathrm{m}}$. Determination of the $\operatorname{sum}\left(x_{1}+s\right)$.]

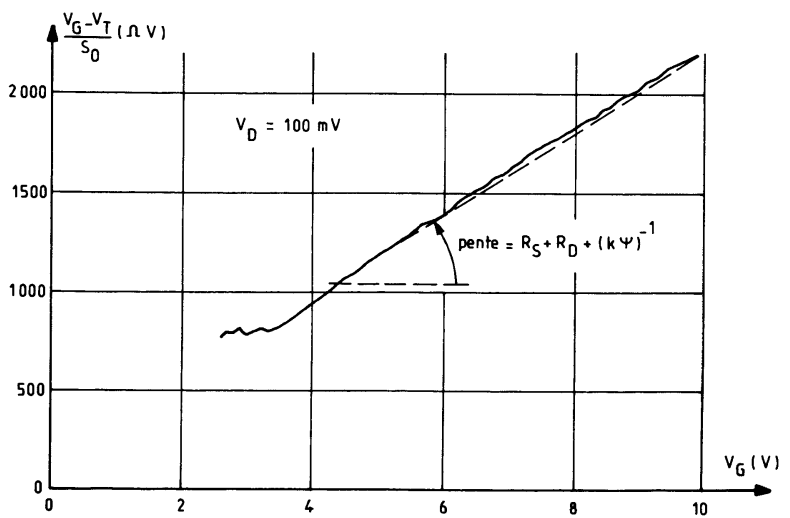

Fig. 5. - Variations de $\frac{V_{\mathrm{G}}-V_{\mathrm{T}}}{S_{0}}$ en fonction de $V_{\mathrm{G}}$. Détermination de $R_{\mathrm{S}}+R_{\mathrm{D}}+(k \cdot \psi)^{-1}$. Mono V.MOS grille polysilicium $\left(L=2 \mu \mathrm{m} ; Z=200 \mu \mathrm{m} ; x_{0}=1500 \AA\right.$; $\left.N_{\mathrm{A}}=3 \times 10^{16} \mathrm{~cm}^{-3}\right)$.

[Determination of the slope $P \equiv R_{\mathrm{S}}+R_{\mathrm{D}}+1 / k \psi$.]
2.2 LE POTENTIEL DE RÉDUCTION DE MOBILITÉ $\psi$ ET LA SOMME DES RÉSISTANCES $R_{\mathrm{S}}+R_{\mathrm{D}}$. - On utilise les mêmes types de transistors décrits précédemment pour déterminer les paramètres $\psi$ et $R_{\mathrm{D}}+R_{\mathrm{S}}$. On trace les variations de la quantité $\left(V_{\mathrm{G}}-V_{\mathrm{T}}\right) / S_{0}$ (obtenue en mesurant la conductance $S_{0}$ ) en fonction de la tension de grille $V_{\mathrm{G}}$. Ces variations linéaires (Fig. 5) ont une pente $P$ (cf. relation 10) égale à :

$$
P_{i}=R_{\mathrm{S}}+R_{\mathrm{D}}+\left(k_{i} \psi\right)^{-1} .
$$

En reportant pour chacun des transistors $i$, la valeur mesurée de la pente $P_{i}$ en fonction des valeurs de la quantité $k_{i}^{-1}$, obtenues au paragraphe précédent, on obtient une droite. Sa pente est égale à $1 / \psi$ et son ordonnée à l'origine vaut $\left(R_{\mathrm{D}}+R_{\mathrm{S}}\right)$. La figure 6 illustre un exemple d'obtention de ces quantités $\psi$ et $R_{\mathrm{S}}+R_{\mathrm{D}}$ par utilisation d'un motif de trois transistors.

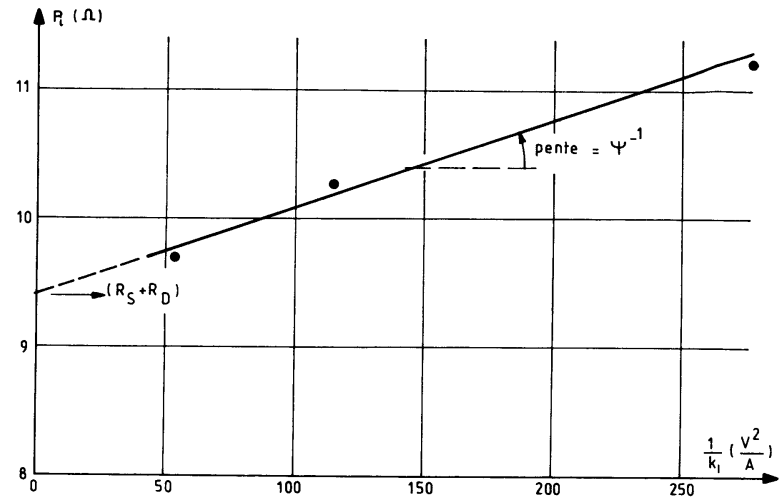

Fig. 6. - Variations de la pente $P$ en fonction du coefficient $1 / k_{i}$. Détermination de $R_{\mathrm{S}}+R_{\mathrm{D}}$ et de $\psi$. Utilisation de trois transistors MOS plan à jonctions diffusées à grille en aluminium $\left(Z=2 \mathrm{~mm} ; x_{0}=1000 \AA ; \quad N_{\mathrm{A}}=1,5 \times\right.$ $10^{16} \mathrm{~cm}^{-3}$ ).

[Experimental variations of the slope $P$ as a function of the coefficient $1 / k_{i}$ for three transistors with different channel length. Determination of the mobility roll-off coefficient $\psi$ and the sum of the series resistances $\left(R_{\mathrm{S}}+R_{\mathrm{D}}\right)$.]

2.3 LA DIFFÉRENCE DES RÉSISTANCES $R_{\mathrm{D}}-R_{\mathrm{S}}$ - Le principe d'obtention de la différence des résistances de drain et de source $\left(R_{\mathrm{D}}-R_{\mathrm{S}}\right)$ est basé sur le tracé des caractéristiques courant-tension en zone ohmique, à tension source-substrat nulle, dans les conditions suivantes :

i) configuration normale du transistor,

ii) configuration inverse, à électrodes de source et de drain inversées.

Ces caractéristiques sont généralement différentes puisque les résistances $R_{\mathrm{D}}$ et $R_{\mathrm{S}}$ ne sont, en principe, pas égales. La figure 7 est un exemple de relevé expérimental des caractéristiques de sortie d'une structure V.MOS, à électrodes de source et de substrat distinctes, dans ces deux configurations.

Théoriquement, les courants de drain en montage 


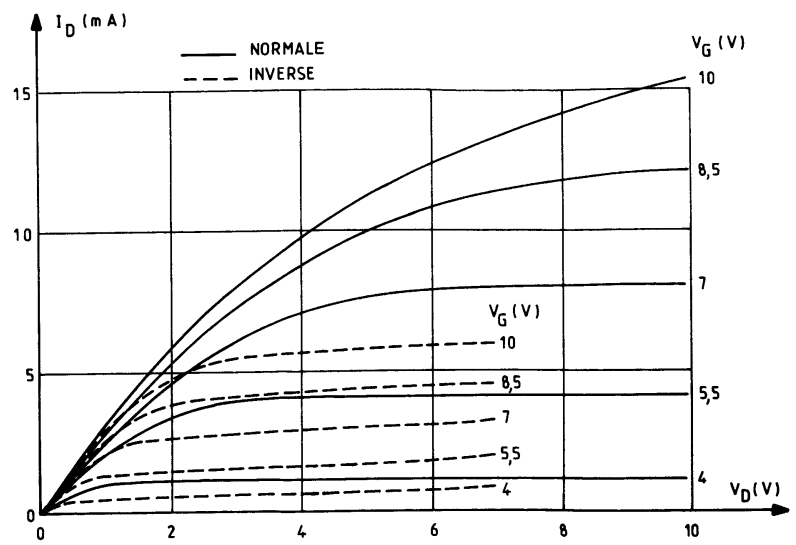

Fig. 7. - Caractéristiques de sortie $I_{\mathrm{D}}\left(V_{\mathrm{D}}\right)$ en configuration normale et inverse Mono $\mathrm{V}$. MOS à grille polysilicium $\left(L=2 \mu \mathrm{m} ; Z=200 \mu \mathrm{m} ; x_{0}=1500 \AA ; N_{\mathrm{A}} \simeq 3 \times\right.$ $\left.10^{16} \mathrm{~cm}^{-3}\right)$.

[Experimental output current-voltage characteristics in the normal and reverse configuration.]

normal et inverse sont régis par la relation 1 dans laquelle les tensions internes de drain $V_{D}^{\prime}$ et de source $V_{\mathrm{R}}^{\prime}$ doivent être respectivement prises égales à $V_{\mathrm{D}}-$ $R_{\mathrm{D}} I_{\mathrm{D}}$ et $R_{\mathrm{S}} I_{\mathrm{D}}$ à l'endroit, puis à $V_{\mathrm{D}}-R_{\mathrm{S}} I_{\mathrm{D}}$ et $R_{\mathrm{D}} I_{\mathrm{D}}$ à l'envers. En restant en zone ohmique (non saturée) on peut montrer (cf. Annexe) que les tensions de grille qu'il faut appliquer dans les deux configurations normale et inverse afin que le composant transite le même courant drain $I_{\mathrm{D}}$, pour la même tension drain $V_{\mathrm{D}}$, sont telles que leur différence $\Delta V_{\mathrm{G}}$ est liée à la différence des valeurs des résistances par la relation approchée :

$$
\begin{aligned}
R_{\mathrm{S}}-R_{\mathrm{D}} & \simeq \frac{\Delta V_{\mathrm{G}}}{I_{\mathrm{D}}} \times \\
\times & {\left[1+\frac{\sqrt{\phi_{\mathrm{B}} \cdot\left(V_{\mathrm{D}}+2 \phi_{\mathrm{F}}\right)}-\sqrt{\phi_{\mathrm{B}} \cdot 2 \phi_{\mathrm{F}}}}{V_{\mathrm{D}}-\left(R_{\mathrm{S}}+R_{\mathrm{D}}\right) \cdot I_{\mathrm{D}}}\right]^{-1} . }
\end{aligned}
$$

Le domaine de validité de cette relation est conditionné par les inégalités suivantes :

$$
\begin{aligned}
\psi & \gg V_{\mathrm{G}}-V_{\mathrm{T}}, \\
V_{\mathrm{D}} & \ll V_{\mathrm{G}}-V_{\mathrm{T}}, \\
2 \phi_{\mathrm{F}} & \gg\left(R_{\mathrm{S}} \text { ou } R_{\mathrm{D}}\right) \cdot I_{\mathrm{D}},
\end{aligned}
$$

qui sont à respecter dans la mise en œuvre expérimentale. Cette dernière consiste à mesurer la translation $\Delta V_{\mathrm{G}}$ que l'on observe sur les deux caractéristiques de transfert $I_{\mathrm{D}}\left(V_{\mathrm{G}}\right)$ relevées (Fig. 8), pour la même tension $V_{\mathrm{D}}$, en configuration normale et inverse, puis à calculer la différence $R_{\mathrm{S}}-R_{\mathrm{D}}$ par la relation 17 . Rappelons, pour ce faire, que les termes $\phi_{\mathrm{B}}, \phi_{\mathrm{F}}$, $R_{\mathrm{S}}+R_{\mathrm{D}}$ ont déjà été déterminés et que les quantités $V_{\mathrm{D}}, I_{\mathrm{D}}$ et $\Delta V_{\mathrm{G}}$ sont mesurées. Notons que le bien-fondé

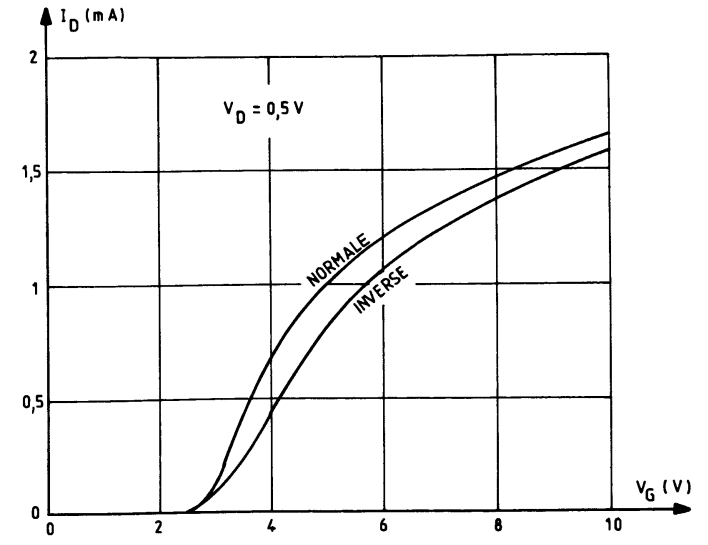

Fig. 8. - Caractéristiques de transfert en configuration normale et inverse Mono V. MOS à grille en polysilicium $V_{\mathrm{D}}=0,5 \mathrm{~V} .\left(L=2 \mu \mathrm{m} ; Z=200 \mu \mathrm{m} ; x_{0}=1500 \AA\right.$; $N_{\mathrm{A}} \simeq 3 \times 10^{16} \mathrm{~cm}^{-3}$.)

[Experimental transfer characteristic in the normal and reverse configuration.]

de cette relation 17 peut être vérifié expérimentalement en utilisant un composant de géométrie symétrique que l'on peut électriquement dissymétriser en introduisant une résistance extérieure en série avec l'une des électrodes de source et de drain.

\section{Conclusion.}

Nous venons de décrire dans cet article les principes de détermination des principaux paramètres des transistors MOS. Les méthodes proposées sont simples de mise en ouvre et utilisables pour toutes les technologies de transistors MOS. Pour pouvoir les appliquer dans leur intégralité, il est nécessaire de disposer de transistors ayant un contact de substrat accessible ainsi que d'au moins deux transistors ayant des géométries identiques sauf en ce qui concerne la longueur de canal.

Les paramètres de mobilité, la longueur effective de canal, la somme de résistances de source et de drain sont déterminés en exploitant les propriétés de la conductance de sortie des structures de test citées précédemment. La différence des résistances de source et de drain est déduite des propriétés des caractéristiques courant-tension lorsque les composants fonctionnent à «l'endroit » ou « à l'envers ».

Le traitement des mesures qu'il faut faire pour accéder aux paramètres est suffisamment simple pour être mis en œuvre manuellement. Dans le cadre d'une caractérisation plus systématique, l'automatisation de ces mesures et leur traitement [8] par un minicalculateur sont aisées.

Sur un plan plus général, la procédure proposée est la seule, à notre connaissance qui permette d'obtenir autant de paramètres et surtout les valeurs séparées des résistances de source et de drain. La détermination 
de ces dernières valeurs est particulièrement importante dans i) le cadre de la caractérisation des résistances des zones dites « drift » des structures D.MOS ou V.MOS devant fonctionner en hautes tensions ii) la connaissance des résistances propres ou des résistances de contact - métal-semiconducteur liées aux nouveaux métaux ou alliages mis en œuvre dans les technologies MOS modernes [9].

\section{Remerciements.}

Les auteurs remercient le personnel de la Centrale de Technologie du L.A.A.S. et plus particulièrement Monsieur C. Solano (fabrication des masques) et Madame F. Rossel (étapes technologiques) pour la réalisation des structures des transistors MOS, support expérimental de cette étude.

\section{Annexe : détermination de la différence $\boldsymbol{R}_{\mathrm{S}}-\boldsymbol{R}_{\mathrm{D}}$.}

Deux approches sont utilisables pour obtenir la différence $R_{\mathrm{S}}-R_{\mathrm{D}}$ :

1) La première est rigoureuse, et consiste à égaler pour la même tension drain $V_{\mathrm{D}}$, les courants $I_{\mathrm{D}}$ en configuration normale - drain au drain - ou inverse - source au drain. Les courants sont égaux lorsque les deux tensions de grille correspondantes $V_{\mathrm{G}_{1}}$ et $V_{\mathrm{G}_{2}}$ sont telles que la relation suivante soit satisfaite :

$$
\begin{aligned}
& \psi \cdot \log \frac{\left(V_{\mathrm{G}_{1}}^{\prime}+\psi-2 \phi_{\mathrm{F}}-V_{\mathrm{D}}+R_{\mathrm{D}} I_{\mathrm{D}}\right) \cdot\left(V_{\mathrm{G}_{2}}^{\prime}+\psi-2 \phi_{\mathrm{F}}-R_{\mathrm{D}} I_{\mathrm{D}}\right)}{\left(V_{\mathrm{G}_{1}}^{\prime}+\psi-2 \phi_{\mathrm{F}}-R_{\mathrm{S}} I_{\mathrm{D}}\right) \cdot\left(V_{\mathrm{G}_{2}}^{\prime}+\psi-2 \phi_{\mathrm{F}}-V_{\mathrm{D}}+R_{\mathrm{S}} I_{\mathrm{D}}\right)}+ \\
& 2 \sqrt{\phi_{\mathrm{B}}} \cdot\left[\sqrt{V_{\mathrm{D}}-R_{\mathrm{D}} I_{\mathrm{D}}+2 \phi_{\mathrm{F}}}-\sqrt{V_{\mathrm{D}}-R_{\mathrm{S}} I_{\mathrm{D}}+2 \phi_{\mathrm{F}}}+\sqrt{R_{\mathrm{D}} I_{\mathrm{D}}+2 \phi_{\mathrm{F}}}-\sqrt{R_{\mathrm{S}} I_{\mathrm{D}}+2 \phi_{\mathrm{F}}}\right. \\
& -\sqrt{V_{\mathrm{G}_{1}}^{\prime}+\psi} \cdot\left[\operatorname{Argth} \sqrt{\left.\frac{V_{\mathrm{D}}-R_{\mathrm{D}} I_{\mathrm{D}}+2 \phi_{\mathrm{F}}}{V_{\mathrm{G}_{1}}^{\prime}+\psi}-\operatorname{Argth} \sqrt{\frac{R_{\mathrm{S}} I_{\mathrm{D}}+2 \phi_{\mathrm{F}}}{V_{\mathrm{G}_{1}}^{\prime}+\psi}}\right]}\right. \\
& \left.+\sqrt{V_{\mathrm{G}_{2}}^{\prime}+\psi} \cdot\left[\operatorname{Argth} \sqrt{\frac{V_{\mathrm{D}}-R_{\mathrm{S}} I_{\mathrm{D}}+2 \phi_{\mathrm{F}}}{V_{\mathrm{G}_{2}}^{\prime}+\psi}}-\operatorname{Argth} \sqrt{\frac{R_{\mathrm{D}} I_{\mathrm{D}}+2 \phi_{\mathrm{F}}}{V_{\mathrm{G}_{2}}^{\prime}+\psi}}\right]\right] \equiv 0 .
\end{aligned}
$$

Dans cette relation $\psi, \phi_{\mathrm{F}}, \frac{Q_{\mathrm{ss}}}{C_{\mathrm{ox}}}-\phi_{\mathrm{MS}}, \phi_{\mathrm{B}}$ sont connus (cf. paragraphes 1 et 2), $V_{\mathrm{G}_{1}}, V_{\mathrm{G}_{2}}, V_{\mathrm{D}}, I_{\mathrm{D}}$ sont mesurés. Compte tenu du fait que la somme $R_{\mathrm{S}}+R_{\mathrm{D}}$ peut être déterminée par la méthode du paragraphe 2.2, la relation $\mathrm{A}$. 1 devient une équation à une inconnue $R_{\mathrm{S}}$ ou $R_{\mathrm{D}}$. Elle ne peut être résolue que numériquement.

2) La deuxième est approchée : elle consiste à développer l'expression $\mathrm{A} .1$ au $2^{\mathrm{e}}$ ordre en considérant que les conditions de mesure sont telles que les inégalités suivantes soient applicables :

$$
\begin{array}{rlrl}
R_{\mathrm{D}} I_{\mathrm{D}} & \text { et } & R_{\mathrm{S}} I_{\mathrm{D}} & \ll 2 \phi_{\mathrm{F}}, \\
V_{\mathrm{D}} & \ll V_{\mathrm{G}}-V_{\mathrm{T}}, \\
\psi & \gg V_{\mathrm{G}}-V_{\mathrm{T}} .
\end{array}
$$

On obtient successivement dans une première phase :

$$
\begin{aligned}
& V_{\mathrm{G}_{1}}^{\prime} \cdot\left(V_{\mathrm{D}}-A I_{\mathrm{D}}\right)- \frac{1}{2}\left(V_{\mathrm{D}}-A I_{\mathrm{D}}\right) \cdot\left(V_{\mathrm{D}}+A I_{\mathrm{D}}-2 R_{\mathrm{D}} I_{\mathrm{D}}+4 \phi_{\mathrm{F}}\right)- \\
&= V_{\mathrm{G}_{2} \cdot\left(V_{\mathrm{D}}-A \phi_{\mathrm{D}}\right)-}^{\prime / 2} \cdot\left[\left(V_{\mathrm{D}}-R_{\mathrm{D}} I_{\mathrm{D}}+2 \phi_{\mathrm{F}}\right)^{3 / 2}-\left(A I_{\mathrm{D}}-R_{\mathrm{D}} I_{\mathrm{D}}+2 \phi_{\mathrm{F}}\right)^{3 / 2}\right]= \\
& \frac{2}{3} \cdot \phi_{\mathrm{B}}^{1 / 2} \cdot\left[\left(V_{\mathrm{D}}-A I_{\mathrm{D}}+R_{\mathrm{D}} I_{\mathrm{D}}+2 \phi_{\mathrm{F}}\right)^{3 / 2}-\left(R_{\mathrm{D}} I_{\mathrm{D}}+2 \phi_{\mathrm{F}}\right)^{3 / 2}\right]
\end{aligned}
$$

avec $A=R_{\mathrm{S}}+R_{\mathrm{D}}$ puis encore :

$$
R_{\mathrm{S}}-R_{\mathrm{D}}=\frac{V_{\mathrm{G}_{1}}-V_{\mathrm{G}_{2}}}{I_{\mathrm{D}}} \cdot\left[1+\frac{\sqrt{\phi_{\mathrm{B}} \cdot\left(V_{\mathrm{D}}+2 \phi_{\mathrm{F}}\right)}-\sqrt{\phi_{\mathrm{B}} \cdot 2 \phi_{\mathrm{F}}}}{V_{\mathrm{D}}-A \cdot I_{\mathrm{D}}}\right]^{-1} .
$$

\section{Bibliographie}

[1] Peng, K. L., Afromowitz, M. A., An Improved Method to Determine MOSFET Channel Length, IEEE Electron Devices Lett. EDL 3 № 12 (1982) 360-362.
[2] Terada, K., Muta, H., A New Method to Determine Effective MOSFET Channel Length, Japan. J. Appl. Phys. 18 ED 5 (1979) 953-959. 
[3] De la Moneda, F. H., Kotecha, H. N., Shatzkes, M., Measurement of MOSFET Constants, IEEE Electron Devices Lett. EDL 3 № 1 (1982) 10-12.

[4] Chern, J. G. J., Chang, P., Motta, R. F., Godinho, N., A New Method To Determine MOSFET Channel Length, IEEE Electron Devices Lett. EDL 1 No 9 (1980) 170-173.

[5] Suciu, P. I., Johnston, R. L., Experimental Derivation of the Source and Drain Resistance of MOS Transistors, IEEE Trans. Electron Devices ED 27 № 9 (1980) 1846-1848.

[6] Goetzberger, A., Ideal MOS Curves for Silicon, Bell Syst. Tech. J. 45 (1966) 1097.

[7] Van Nielen, J. A., Memelink, O. W., The Influence of the substrate upon the D.C. characteristics of Silicon M.O.S.T., Philips Research Report 22 (1967) $57-71$.
[8] Tranduc, H., Caquot, E., Guégan, G., Rossel, P., M.A.A.C.S.I.M. : Méthodes Automatiques d'Acquisition des Caractéristiques Statiques et d'Identification des paramètres des transistors Métal-Oxyde-Semiconducteur. Note Technique LAAS NT 80.I.21 (1980).

[9] ChOw, Paul, T. S., The development of refractory gate metallisation for VLSI, ch. 4, Ph. D. Thesis. Rensselaer Polytechnic Institute, Troy (N.Y.), May 1982.

[10] Rossel, P., Martinot, H., VassiliefF, G., Accurate two Sections Model for MOS Transistor in saturation, Solid State Electron. 19 (1976) 51-56.

[11] MA, Y. R., WANG, K. L., A New Method to Electrically Determine Effective MOSFET Channel Width, IEEE Trans. Electron Devices, ED 29 no 12 (1982) $1825-1827$ 